Eye (2011) 25, 955-956; doi:10.1038/eye.2011.35; published online 11 March 2011

Sir,

Incidence of post-operative endophthalmitis following 23-gauge transconjunctival sutureless vitrectomy in the United Kingdom: a survey

We carried out a confidential and anonymous 19-question online survey aimed at members registered on the Britain \& Eire Association of Vitreoretinal Surgeons (BEAVRS) online forums, looking at rates of post-operative endophthalmitis following 23-gauge (23G) trans-conjunctival sutureless vitrectomy (TSV).

Participants were asked to state the total number of $23 \mathrm{G}$ TSV cases performed in their career, and the number of cases of post-operative endophthalmitis that occurred from these. Endophthalmitis was not defined. Participants were asked to check surgical logbooks for accuracy before quoting case and complication numbers.

In all, 45/144 members registered on the BEAVRS online forums in April 2009 participated, giving a 31\% response rate. Of the participants, $36 \%$ preferred 23G. Of these, the most popular port system was Alcon-based for $39,52 \%$ had performed a two-step port entry technique, and $87 \%$ had performed a conjunctival sac wash with $5 \%$ povidone-iodine. In addition, $26 \%$ routinely used fluidair exchange to prevent hypotony even when there was no other clinical indication for endotamponade, and $87 \%$ gave antibiotics sub-conjunctivally. A total of $494423 \mathrm{G}$ TSV cases were estimated to have been performed by 23 surgeons. Two cases of post-operative infective endophthalmitis were reported, giving an overall incidence rate of 1 in $2472(0.040 \%)$.

This survey had a low response rate. This is likely due to the fact that the BEAVRS forums are open to membership from ophthalmic trainees and VR surgeons outside the United Kingdom (who were not eligible to participate), that not all UK VR surgeons practice 23G TSV (and therefore may have decided not to participate in the survey), and that some surgeons do not like discussing operative complication rates. Without documentary evidence, the numbers of cases performed as quoted by participants can only be taken as estimates.

Internationally published rates for $20 \mathrm{G}$ vitrectomy range from $0.018 \%{ }^{1}$ to $0.07 \% .^{2}$ The only UK evidence quotes $0.038 \%$. $^{3}$ Therefore, the estimated rate of postoperative endophthalmitis of $0.040 \%$ for UK-based 23G TSV is acceptable when compared with these numbers. Obtaining a more accurate rate would require a prospective national audit with an open reporting system for complications.

\section{Conflict of interest}

The authors declare no conflict of interest.

\section{References}

1 Kunimoto DY, Kaiser RS, Wills Eye Retina Service. Incidence of endophthalmitis after 20- and 25-gauge vitrectomy. Ophthalmology 2007; 114(12): 2133-2137.
2 Cohen SM, Flynn Jr HW, Murray TG, Smiddy WE. Endophthalmitis after pars plana vitrectomy. The Postvitrectomy Endophthalmitis Study Group. Ophthalmology 1995; 102: 705-712.

3 Mollan SP, Mollan AJ, Konstantinos C, Durrani OM, Butler L. Incidence of endophthalmitis following vitreoretinal surgery. Int Ophthalmol 2009; 29(3): 203-205.

KC Patel ${ }^{1}$ and R Rahman ${ }^{2}$

${ }^{1}$ Hull Royal Infirmary, Hull, UK

${ }^{2}$ Calderdale Royal Hospital, Halifax, UK

E-mail: karnesh@doctors.org.uk or

binarahman@fsmail.net

This study was previously presented at BEAVRS 2009, Amsterdam.

Eye (2011) 25, 956; doi:10.1038/eye.2011.46; published online 11 March 2011 Sir, microscopy: a case report

Fungal keratitis (FK) is a severe blinding eye disease and a major cause of ocular morbidity. ${ }^{1}$ The clinical features of FK are not specific and the diagnosis is frequently not suspected until an aggressive treatment for bacterial, viral, or amebic keratitis has failed.

\section{Case report}

We report a case of a 65-year-old woman who developed a severe infectious keratitis in her left eye after a corneal injury caused by a tree branch and was initially treated by her family doctor with a local combination of tobramycin and dexamethasone. After 4 days she presented to an ophthalmology department for decreased vision and increased pain in her left eye. On initial evaluation, best-corrected visual acuity was hand movement in her left eye. Clinical examination revealed numerous purulent secretions, a conjunctival hyperaemia, and a large irregular whitish central corneal infiltrate $(4.5 \mathrm{~mm} \times 5 \mathrm{~mm})$ with $1 \mathrm{~mm}$ hypopyon. The left eye fundus could not be visualized. Examination of her right eye was normal. Corneal scrapings were analysed by direct examination and culture. She was started on hourly topical fortified tobramycin, gentamicin and vancomycin, and oral levofloxacin. After 3 days, presence of Pseudomonas aeruginosa was identified, vancomycin and tobramycin were stopped, and topical fortified ceftazidime was added according to bacterial sensitivity. However, after 5 days of this treatment the corneal infiltrate increased in size and depth, and the hypopyon increased to $2 \mathrm{~mm}$ (Figure 1a). The patient was referred to our department for an in vivo confocal microscopy (IVCM) examination.

Interestingly, IVCM images (Heidelberg Retina Tomograph 3 - Rostock Cornea Module, Heidelberg Engineering, Heidelberg, Germany) of the left eye 

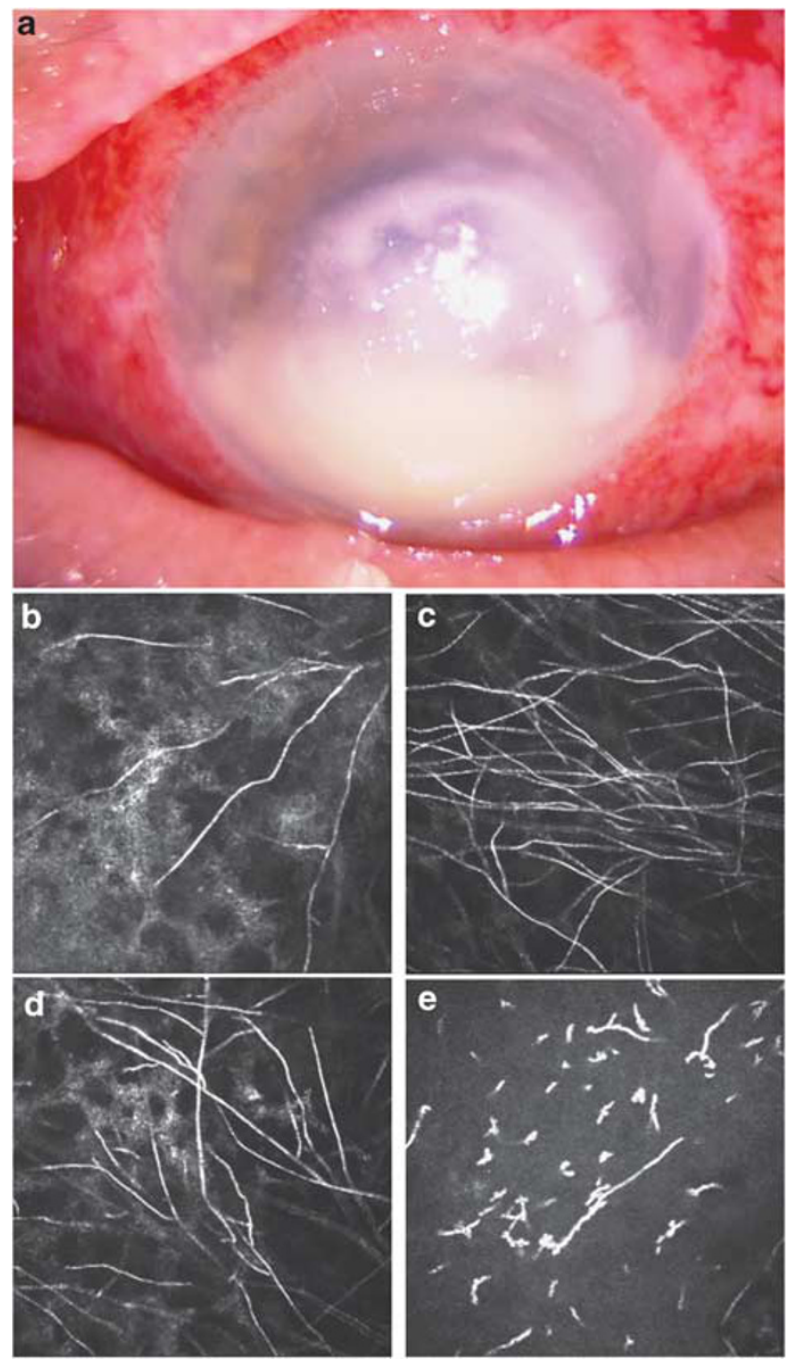

Figure 1 (a) Photography of the left eye showing a large corneal central whitish irregular infiltrate with $2 \mathrm{~mm}$ hypopion. (b-d) In vivo confocal microscopy images $\left(400 \times 400 \mu \mathrm{m}^{2}\right)$ showing numerous hyper-reflective interlocking and branching linear structures, 5-7 $\mu \mathrm{m}$ width and $200-400 \mu \mathrm{m}$ length, possibly corresponding to fungal hyphae. These images were obtained in the stroma at the boundaries of the central corneal infiltrate: (b) in the nasal area, depth: $115 \mu \mathrm{m}$; (c) in the superior area, depth: $223 \mu \mathrm{m}$; (d) in the superior area, depth: $106 \mu \mathrm{m}$. (e) In vivo confocal microscopy image $\left(400 \times 400 \mu \mathrm{m}^{2}\right)$ of the corneal stroma (45 $\mu \mathrm{m}$ depth) showing a stromal oedema with infiltration of inflammatory cells. The stroma appeared as a dark background with hyper-reflective small structures with an occasional dendritic-like pattern corresponding to inflammatory cells.

showed hyper-reflective, thin, and branching interlocking linear structures in the stroma, 5-7 $\mu \mathrm{m}$ in width and $200-400 \mu \mathrm{m}$ in length, possibly corresponding to filamentous fungi (Figure $1 \mathrm{~b}$ and $\mathrm{c}$ and Supplementary video online). These hyper-reflective structures were detected on the boundaries of the central corneal infiltrate at depths varying from 100 to $300 \mu \mathrm{m}$ and were surrounded by oedema and infiltration of inflammatory cells (Figure 1d).
Topical amphotericin B and topical and systemic voriconazole were added to the previous treatment. A penetrating keratoplasty was performed because of a perforation that occurred 5 days later, and the direct examination and culture of the cornea confirmed the presence of a Fusarium sp within the stroma.

\section{Comments}

This case of bacterial and FK demonstrates the usefulness of IVCM for the diagnosis of filamentous fungal keratitis. Initial corneal scrapings were negative for fungi probably because of the localization of the hyphae deeper in the stroma. In such cases, IVCM can visualize filamentous fungi within the whole cornea of patients and may be helpful in managing this disease by providing a rapid diagnosis so that treatment may be initiated earlier. ${ }^{2}$

Fungal hyphae were observed with IVCM at the boundaries of the corneal infiltrate, where a better visualization of corneal structures and probably fungal hyphae is obtained. To carry out this dynamic examination and to enhance the effectiveness of IVCM, it must be performed but also interpreted by an experience operator. ${ }^{2}$ Despite these limitations, IVCM remains a valuable diagnostic modality for FK as recently confirmed by Vaddavalli et al., ${ }^{3}$ who demonstrated in a controlled study a sensitivity of $89.2 \%$ and a specificity of $92.7 \%$ of IVCM for the diagnosis of FK as compared to standard microbiology.

\section{Conflict of interest}

The authors declare no conflict of interest.

\section{References}

1 Thomas PA, Geraldine P. Infectious keratitis. Curr Opin Infect Dis 2007; 20: 129-141.

2 Labbé A, Khammari C, Dupas B, Gabison E, Brasnu E, Labetoulle $\mathrm{M}$ et al. Contribution of in vivo confocal microscopy for the diagnosis and the management of infectious keratitis. Ocul Surf 2009; 7: 41-52.

3 Vaddavalli PK, Garg P, Sharma S, Sangwan VS, Rao GN, Thomas R. Role of confocal microscopy in the diagnosis of fungal and acanthamoeba keratitis. Ophthalmology 2011; 118(1): 29-35.

\section{A Labbé1, $2,3,4,5$, E Gabison ${ }^{6}$, I Cochereau ${ }^{6}$ and C Baudouin ${ }^{1,2,3,4,5}$}

'Department of Ophthalmology,

Quinze-Vingts National Ophthalmology Hospital,

Paris and Ambroise Paré Hospital,

AP-HP, University of Versailles Saint-Quentin en

Yvelines, Versailles, France

${ }^{2}$ INSERM, U968, Paris, France

${ }^{3}$ UPMC University Paris 06, UMR S 968,

Institut de la Vision, Paris, France

${ }^{4}$ CNRS, UMR 7210, Paris, France

${ }^{5}$ Center of Clinical Investigations 503

Quinze-Vingts National Ophthalmology Hospital,

Paris, France 
${ }^{6}$ Department of Ophthalmology, Rothschild

Foundation and Bichat Hospital, AP-HP,

Paris, France

E-mail: antoinelabbe@hotmail.com

Eye (2011) 25, 956-958; doi:10.1038/eye.2011.51; published online 11 March 2011

Supplementary Information accompanies the paper on Eye website (http:/ /www.nature.com/eye)

Sir,

Peer-reviewed publication of abstracts presented at the Royal College of Ophthalmologists (RCOphth) Annual Congress

Approximately $50 \%$ of the abstracts initially presented at scientific meetings will never be published in peer-reviewed journals. ${ }^{1}$ Such unpublished research is therefore not available via electronic databases (eg, MEDLINE) to clinicians or to those undertaking systematic literature reviews.

The Cochrane review ${ }^{1}$ included 79 studies, only three from ophthalmic meetings, all being from North America in the 1980s. We therefore conducted a PubMed literature search using each author from the abstracts of the 'Final Programme and Abstracts' RCOphth Congress 2004 (search performed 61 months after the congress submission closing date). A publication was deemed to relate to the same work as the abstract if at least one author, methodology, or at least one of the conclusions of the study were the same to allow for subsequent continued recruitment. ${ }^{2}$

Of the 179 abstracts, 64 (35.8\%; 95\% CI 29-43\%) reached full publication in peer-reviewed journals, most frequently Eye (23/64) and British Journal of Ophthalmology (18/64). The mean time that elapsed between Congress 2004 and the publication was 20.1 months (95\% CI 16.9-23.4 months) (see Figure 1).

Multivariate analysis of the factors influencing publication revealed that the number of authors showed significant positive correlation with subsequent publication rate: abstracts with only one or two authors (26\% subsequently published) compared with $36 \%$ for 3-5 authors and 50\% for 6-or-more authors.

Other factors analysed failed to show any significant difference in subsequent publication rates; posters only

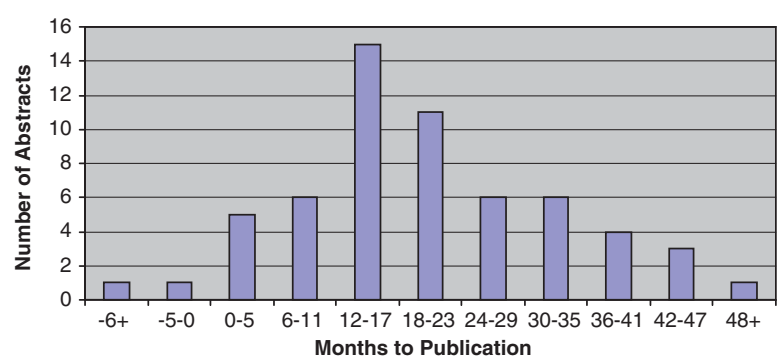

Figure 1 Time elapsed to publication following RCOphth Congress 2004
(35\% later published)/podium presentations (41\%); prospective studies $(33 \%) /$ retrospective $(33.9 \%)$; UK only research $(35 \%)$ /international collaboration $(45 \%)$; basic-science research $(41.7 \%) /$ clinical research $(36.5 \%) /$ service delivery-related studies (26.3\%). Publication bias is a well-recognised phenomenon, but research reporting statistically significant $(44.4 \%)$ and nonsignificant $(43.6 \%)$ results were equally likely to be published.

Lead authors based in a teaching hospital or university were more successful $(39.5 \%)$ in getting papers published than those working in non-academic settings (28.3\%).

Nearly two-thirds of the studies presented at the abstract at the RCOphth Congress 2004 had not been published in peer-reviewed journals more than 5 years after the closing date for submission. Studies with larger numbers of authors were more likely to reach publication, possibly because involvement of more authors may mean that there are more people to scrutinise the design and conduct of the research, review drafts, and improve overall quality.

\section{Conflict of interest}

The authors declare no conflict of interest.

\section{References}

1 Scherer R, Langenberg P, von Elm E. Full publication of results initially presented in abstracts. Cochrane Database Syst Rev 2007; (2): MR000005.

2 Buchan JC, Spokes DM. Do recorded abstracts from scientific meetings concur with the research presented? Eye 2010; 24: 695-698.

\section{JC Buchan1, A Bastawrous², M Aldawoud ${ }^{3}$ and D Shickle ${ }^{4}$}

${ }^{1}$ CBM Ophthalmologist, Kissy UMC Eye Hospital, Freetown, Sierra Leone

${ }^{2}$ Department of Ophthalmology, St Paul's Eye Unit, Royal Liverpool Hospital, Liverpool, UK

${ }^{3}$ Highfield Health Centre, Bradford, UK

${ }^{4}$ Institute of Health Sciences, University of Leeds, Leeds, UK

E-mail: andrew.bastawrous@gmail.com

Eye (2011) 25, 958; doi:10.1038/eye.2011.52; published online 11 March 2011

Sir, Response to 'Spontaneous sub-conjunctival haemorrhage in patients using long-term topical corticosteroids'

I read with interest the article by Mercieca et $a l^{1}$ describing a higher incidence of subconjunctival haemorrhage in a cohort of uveitic patients who were on long-term topical steroid therapy compared with a comparison group of patients using glaucoma medication. Although they describe systemic hypertension as a risk factor for subconjunctival haemorrhage, they did 